\title{
Optimal blood transfusion therapy in haemoglobinopathies
}

\author{
John Porter \\ Professor of Haematology, University College London and Joint Red Cell Unit, UCLH and \\ Whittington Hospitals, London, UK
}

For reasons of time, this short talk will be confined to the optimal frequency, timing, indications and dosing of blood transfusion. Blood transfusion protocols in thalassaemia syndromes are more widely agreed (1) than for sickle disorders but questions still remain about optimal $\mathrm{Hb}$ levels, timing and frequency. In transfusion thalassaemia thalassaemias (TDT), the purpose of blood transfusion is to maximise quality of life by correcting anaemia and suppressing ineffective erythropoiesis, whilst minimising the complications of the transfusion itself. Under-transfusion will limit growth and physical activity while increasing intramedullary and extra-medullary erythroid expansion. Over transfusion may cause unnecessary iron loading and increased risk of extra-hepatic iron deposition however. Although guidelines imply a 'one size fits all' approach to transfusion, in reality this is not be the case. Indeed a flexible approach crafted to the patient's individual requirements and to the local availability of safe blood products is needed for optimal outcomes. For example in $\mathrm{HbE} \beta$ thalassaemias, the right shifted oxygen dissociation curve tends to lead to better oxygen delivery per gram of $\mathrm{Hb}$ than in $\beta$ thalassaemia intermedia with high $\mathrm{Hb}$ F. Patients with E $\beta$ thal therefore tend to tolerate lower $\mathrm{Hb}$ values than $\beta$ thalassaemia intermedia. Guidelines aim to balance the benefits of oxygenation and suppression of extra-medullary expansion with those of excessive iron accumulation from overtransfusion. In an Italian TDT population, this balance was optimised with pre-transfusion values of $9.5-10.5 \mathrm{~g} / \mathrm{dl}$ (2). However this may not be universally optimal because of different levels of endogenous erythropoiesis with different genotypes in different populations. Recent work by our group (3) suggests that patients with higher levels of endogenous erythropoiesis, marked by higher levels of soluble transferrin receptors, at significantly lower risk of cardiac iron deposition than in those where endogenous erythropoiesis is less active, as would be the case in transfusion regimes achieving higher levels of pre-transfusion $\mathrm{Hb}$.

In sickle cell disorders, the variability in the phenotype between patients and also within a single patient at any given time means that the need for transfusion also varies. A consideration in sickle disorders, not usually applicable to thalassaemia syndromes, is that of exchange transfusion versus simple top up transfusion. Exchanges have the advantages of lower iron loading rates and more rapid lowering of $\mathrm{HbS} \%$. Disadvantages of exchange transfusion are of increased exposure to blood products with inherent increased risk of allo-immunisation or infection, requirement for better venous access for adequate blood flow, and requirements for team of operators capable of performing either manual or automated apheresis, often at short notice. Some indications for transfusion in sickle disorders are backed up by randomised controlled data, such as for primary and secondary stroke prevention, or prophylaxis of sickle related complications for high-risk operations (4). Others are widely practiced as standard of care without randomised data, such as treatment of acute sickle chest syndrome. Other indications for transfusion, not backed up by randomised studies, but still widely practiced in selected cases, include the management of pregnancy, leg ulceration or priapism and repeaed vaso-occlusive crises. Allo-immunisation is more common in sickle patients than in thalassaemia disorders and hyper-haemolysis is a rare but growing serious problem in sickle disorders. It is arguable that increased use of transfusion early in life, is indicated to decrease silent stroke rates and that early exposure to blood will decease red cell allo-immunisation rates.

\section{References}

1. Cappellini, MD, Cohen, A, Porter, J, Taher, A, Viprakasit, V. TIF Guidelines for the Management of Transfusion Dependent Thalassaemia (TDT) Nicosia (CY) (2014).

2. Cazzola $\mathrm{M}$, et al. A moderate transfusion regimen may reduce iron loading in beta-thalassemia major without producing excessive expansion of erythropoiesis. Transfusion;37:135-40 (1997).

3. Garbowski MV, et al. Residual erythropoiesis protects against myocardial hemosiderosis in transfusion-dependent thalassemia by lowering labile plasma iron via transient generation of apotransferrin Haematologica 102(10) 1640-1649 2017).

4. De Baun MR, et al., Controlled trial of transfusions for silent cerebral infarcts in sickle cell anemia N Engl J Med; 371:699710. (2014).

\author{
Correspondence: John Porter, Haematology, University College \\ London and Joint Red Cell Unit, UCLH and Whittington Hospitals, \\ London, UK \\ This work is licensed under a Creative Commons Attribution 4.0 \\ License (by-nc 4.0). \\ CC Copyright J. Porter, 2018 \\ Licensee PAGEPress, Italy \\ Thalassemia Reports 2018; 8:7477 \\ doi:10.4081/thal.2018.7477
}

